\title{
Nitrite, an Electron Donor for Anoxygenic Photosynthesis
}

\section{Benjamin M. Griffin, ${ }^{*}$ Joachim Schott, Bernhard Schink}

A lthough compounds of the sulfur cycle, and more recently the iron cycle, are well studied electron donors for anoxygenic photosynthesis, no analogous oxidations in the ni trogen cycle are known. We report a previously unknown process in which anoxygenic photo trophic bacteria use nitrite as an electron donor for photosynthesis, providing a microbial mechanism for the stoichiometric oxidation of nitrite to nitrate in the absence of oxygen. To examine nitrite as a possible electron donor for anoxygenic photo trophs, we established enrichment cultures derived

oxidizing cultures, suggesting that nitrate did not form because of a combination of oxygenic photosynthesis and aerobic nitrification. No growth or nitrite oxidation occurred in cultures incubated in the dark or in uninoculated bottles, thereby ruling out the possibilities that nitrate was produced by anaerobic ammonia oxidation (anammox) or abiotic, photochemical processes.

Light dark shift experiments performed over several days with enrichment cultures transferred five times showed that growth and nitrate produc tion depended on both light and nitrite (Fig. 1). roseopersicina (98\% identical). Thiocapsa spe cies are widely distributed purple sulfur bacteria of the order Chromatiales and are metabolic gen eralists capable of photoautotrophic growth on a variety of common inorganic electron donors, in ad dition to aerobic chemolithoautotrophic growth (3).

Although phototrophs are known to directly influence the nitrogen cycle through reductive processes such as nitrogen fixation, assimilation, and respiration (4), this is the only example of a photosynthetically driven oxidation in the nitro gen cycle. In principle, this photosynthetic pro cess could compete for nitrite in the environment with other key nitrogen cycle processes such as denitrification, aerobic nitrification, or anammox.

In 1970, Olson proposed in detail how the water oxidizing activity of oxygenic photosynthe sis may have evolved from anoxygenic photo synthesis through a series of inorganic nitrogen electron donors with increasing midpoint potentials (5). The nitrite nitrate couple, with a standard redox

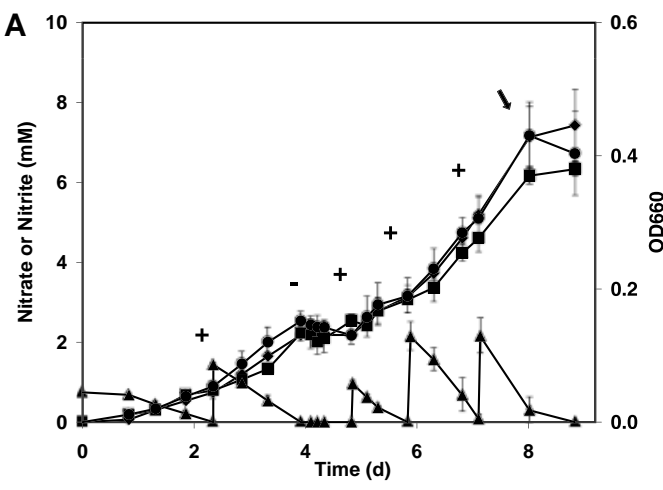

Fig. 1. Time courses for nitrite consumed $(\boldsymbol{\Delta})$, nitrate produced $(\boldsymbol{\nabla})$, cumulative nitrite consumed $(\diamond)$, and growth as the change in optical density $\left(\Delta \mathrm{OD}_{660}\right)(\bullet)$ for triplicate enrichment cultures $(N=3)$. Data are mean \pm SD. (A) Initially incubated in the light. (B) Initially incubated in the dark. The plus signs indicate nitrite feedings, and arrows denote a switch from the initial light condition. The minus signs indicate when the cultures were starved of nitrite to assess nitrite dependence of growth. (C) Phase-contrast micrograph of strain KS. The scale bar represents $10 \mu \mathrm{m}$.
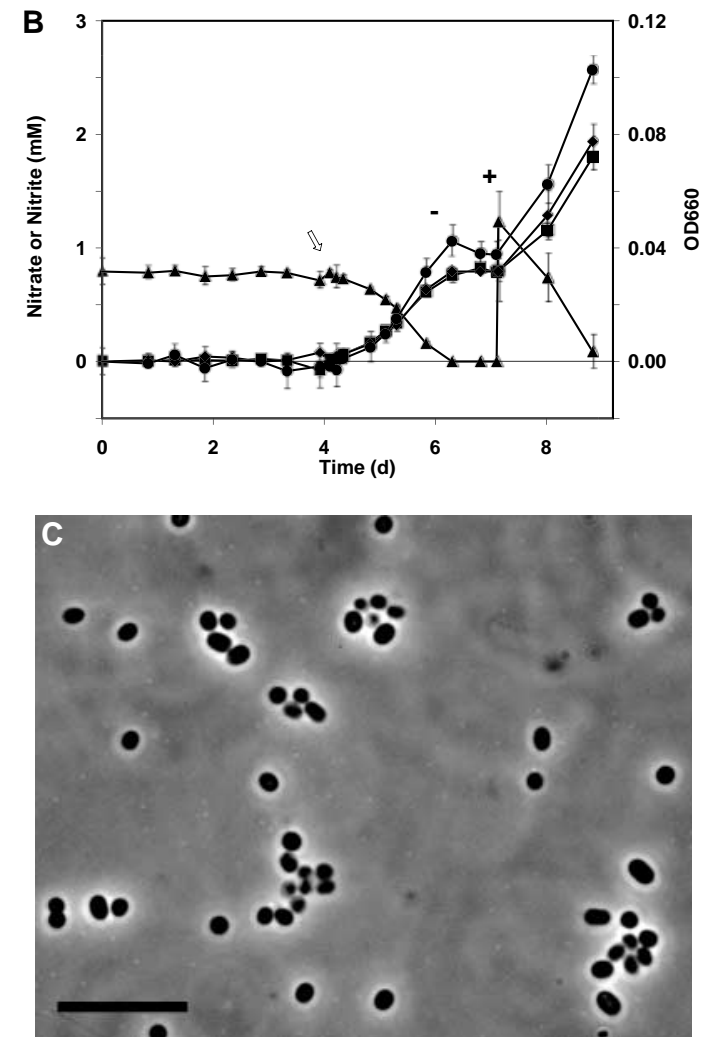
potential of $+0.43 \mathrm{~V}$, could theo retically donate electrons to the quinone type reaction center in pur ple sulfur bacteria, where the bac teriochlorophyll primary donor has a midpoint potential as high as $+0.49 \mathrm{~V}(6)$. This work demon strates nitrite as the highest potential electron donor for anoxygenic photo synthesis known so far and provides a modern example of an electron donor once implicated in the evo lution of oxygenic photosynthesis.

References and Notes

1. Materials and methods are available on Science Online.

2. J. F. Imhoff, in Anoxygenic Photosynthetic Bacteria, R. E. Blankenship, M. T. Madigan, C. E. Bauer, Eds. (Kluwer, Dordrecht, Netherlands, 1995), pp. 115.

3. J. F. Imhoff, in The Prokaryotes, M. Dworkin et al., Eds. (Springer Verlag, New York, ed. 3, 2006), vol. 6, pp. 846873.

4. J. P. Megonigal, M. E. Hines, P. T. Visscher, in Treatise on Geochemistry, vol. 8, W. H. Schlesinger, Ed. (Elsevier, Amsterdam, 2003), pp. 317424.

5. J. M. Olson, Science 168, 438 (1970).

from local sewage sludge and several freshwater sediments in anoxic, bicarbonate buffered mineral medium (1). Low amounts of nitrite (1 to $2 \mathrm{mM}$ ) were fed repeatedly to avoid toxicity, and the cultures were incubated continuously in the light.

After incubating in the light for several weeks, enrichment cultures from 10 out of 14 sampling sites oxidized nitrite to nitrate and de veloped pink coloration, as typical of anoxygenic phototrophs. Absorption spectra of intact cells revealed maxima at $799 \mathrm{~nm}$ and $854 \mathrm{~nm}$, which are characteristic of bacteriochlorophyll a (2). No chlorophyll a or oxygen was observed in nitrite
The rate of nitrite consumption increased on mul tiple feedings and approached $2 \mathrm{mM}$ per day after 1 week in the light. As expected for a photoauto trophic process, nitrite consumed, nitrate produced, and biomass formed were all tightly correlated; $\mathrm{ni}$ trate was formed from nitrite near stoichiometrically.

We isolated the numerically dominant coccus ( 2 to $3 \mu \mathrm{m}$ in diameter) from the most active en richment culture derived from Konstanz sewage sludge by dilution to extinction in liquid medium (Fig. 1C) (1). Analysis of the $16 S$ ribosomal RNA gene sequence revealed that the strain, des ignated $\mathrm{KS}$, is most closely related to Thiocapsa
6. M. A. Cusanovich, R. G. Bartsch, M. D. Kamen, Biochim. Biophys. Acta 153, 397 (1968).

\section{Supporting Online Material}

www.sciencemag.org/cgi/content/full/316/5833/1870/DC1 Materials and Methods

References

3 January 2007; accepted 19 April 2007 10.1126/science. 1139478

Department for Biology, Universität Konstanz, D 78457 Konstanz, Germany.

*Present address: Institute for Genomic Biology, University of Illinois, Urbana, IL 61801, USA. To whom correspondence should be addressed. E mail: griff113@uiuc.edu 